\title{
MOTIVATION AND EMOTIONS IN EFFECTIVE EATING HABITS CHANGE. MULTI-METHOD EXPLORATORY STUDY
}

\author{
Grażyna Wąsowicz ${ }^{1}$, Magdalena Poraj-Weder ${ }^{1}, \&$ Christophe Matthys ${ }^{2,3}$ \\ ${ }^{I}$ Faculty of Psychology, University of Warsaw, Warsaw (Poland) \\ ${ }^{2}$ Nutrition \& Obesity, Clinical and Experimental Endocrinology, Department of Chronic Diseases, \\ Metabolism and Aging, KU Leuven (Belgium) \\ ${ }^{3}$ Clinical Nutrition, Department of Endocrinology, University Hospitals Leuven (Belgium)
}

\begin{abstract}
Many studies have shown that attempts to change one's behaviour often end in failure. Old habits reassert themselves and new attempts to alter them are necessary, leading to frustration and disappointment.

The aims of the study were to explore within one theoretical framework the importance of motivation and emotion in the process of changing eating habits, and to estimate the role of health professionals in the process. The research employed a multi-method approach, and quantitative and qualitative studies were conducted. The main goal of the quantitative study 1 . was to assess the role of health professionals in achieving effective change and to widen the knowledge of psychological (motivational and emotional) determinants of the effectiveness of eating habits change. The purpose of the quantitative study 2 . was to deepen the knowledge acquired in the first study. A total of 420 men and women undergoing the process of change were recruited to participate in the first study. Motivation was measured by Treatment Self-Regulation Questionnaire (Diet) (Ryan \& Connell, 1989; Levesque, Williams, Elliot, Pickering, Bodenhamer et al., 2007), emotions experienced in the process were assessed by Scale of Positive and Negative Experience (Diener \& Biswas-Diener, 2009) and emotional attitude towards a process of change - by Scale on Emotions Related to the Behaviour Change, created for the purpose of the study. Three measures of declared effectiveness of diet change were applied: objective (change in weight in kilograms) and subjective (evaluation of dietary change into more healthful food consumption and satisfaction with self-efficacy in the process). Study 2. was conducted using individual, semi-structured, in-depth interviews (IDIs) (Angrosino, 2010; Kvale, 2010). Two groups of respondents were examined: 6 individuals changing their eating habits and 7 health professionals. The thematic analysis (TA; Braun \& Clark, 2006; Clark et al., 2015) deductive method was used to analyze collected data. Results from both studies show that emotional attitudes toward dietary changes and emotional experiences during the process of change - neglected thus far in empirical studies - are crucial for the effectiveness of the process. Moreover, the emotional aspects modify the relationship between different types of motivation (as defined within the Self-Determination Theory) and the effectiveness of the process.
\end{abstract}

Keywords: Weight loss, motivation, emotions, health professionals, patients, self-determination theory. 